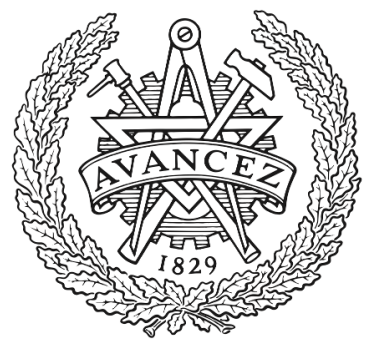

CHALMERS

UNIVERSITY OF TECHNOLOGY

\title{
Comparisons of Concentrated and Distributed Winding PMSM in MV Power Generation
}

Downloaded from: https://research.chalmers.se, 2023-04-26 09:40 UTC

Citation for the original published paper (version of record):

Zhao, J., Liu, Y., Xu, X. (2018). Comparisons of Concentrated and Distributed Winding PMSM in

MV Power Generation. 2018 XIII International Conference on Electrical Machines (ICEM):

2437-2443. http://dx.doi.org/10.1109/ICELMACH.2018.8507199

N.B. When citing this work, cite the original published paper. 


\title{
Comparisons of Concentrated and Distributed Winding PMSM in MV Power Generation
}

\author{
J. Zhao, Y. Liu and X. Xu
}

\begin{abstract}
This paper presents the design and comparison of an interior permanent magnet synchronous generator (IPMSG) with fractional-slot concentrated winding (FSCW) and distributed overlap winding used in medium voltage (MV) medium power generator. Analytical method is used to evaluate the combination of slots and poles taking into consideration winding factor and magnetic motive force (MMF). The impact of rotor skewing on slot harmonics reduction is presented mathematically and later verified using finite element method (FEM) simulation. The electromagnetic performance of the two generators are compared using efficiency, thermal and no load induced voltage. Radial electromagnetic forces on tooth and airgap are calculated to assess vibration characteristics of the two machines. Furthermore, the insulation requirements are compared between concentrated and distributed windings using electrical fields generated in stator slot between winding layers. It is observed that both type of windings gives similar performance with concentrated winding providing better ease of insulation.
\end{abstract}

Index Terms-Permanent magnet machine, electromagnetic analysis, power generation, harmonic analysis, finite element analysis, high voltage techniques, insulation, energy efficiency.

\section{INTRODUCTION}

$\mathrm{F}$ acing the challenge of energy crisis and environmental pollution, renewable energy becomes more and more a popular research area. Among the available renewable energy sources, tidal power is considered to be promising due to its concentrated energy density. Generator plays an important role in energy conversion. Because of its offshore work condition, high power density, high efficiency and high reliability is expected. A comparison of different type of generators has been made in [1] and it shows that permanent magnet synchronous generator is a better choice for tidal power generation. High power generating systems are suffering from the large size of cable which increases the system cost and decreases system power density, due to high current.

Higher voltage is a good way to solve the system problem. But insulation of the winding in generator becomes an issue. Traditionally, medium voltage electrical machine applied in industrial area mainly use distributed overlap winding. However, the multilayer insulation and long end-winding decrease the power density of such generators. Fractionalslot concentrated winding (FSCW) provides an alternative where every coil is wrapped on a tooth. No overlap in the end winding can reduce the insulation volume of end winding and may increase the power density. So, concentrated winding machine becomes a suitable candidate

This work was supported by the European Union's Horizon 2020 research and innovation program under Grant 654438 .

J. Zhao, Y. Liu and X. Xu are with the division of Electric Power Engineering, department of Electrical Engineering, Chalmers University of Technology, Gothenburg, 41296 Sweden (email: zjian@chalmers. se, yujing.liu@chalmers. se, xiangdong.xu@chalmers. se) for medium voltage application.

Because of the distribution arrangement of coils, integral slot distributed winding machine has more sinusoidal flux distribution. Only several harmonics generated by slot open add to the fundamental. On the contrary, concentrated winding's coil is wrapped on one tooth, the flux distribution is non-sinusoidal. And it taken a certain harmonic as its fundamental wave, this leading to more field harmonics especially low order harmonic in the airgap. In recent few years, the benefit of concentrated winding is studied in many researches [2], [3]. Its cogging torque performance, torque ripple character is analyzed in [4 to 6]. The disadvantages also caught many researcher's eyes. Its high core loss, high magnet loss and peculiar noise and vibration performance are hot issues in its application [7 to 10].

The advantage and drawback confine the use of concentrated winding in low power and low voltage area, especially servo motor. Applied in medium voltage, the insulation capacity is taken in the first place. Due to its special structure, concentrated winding has a better insulation, but the performance is not so clear. This paper presents a comparative design and the evaluation of machine with distributed overlap winding and fractional-slot concentrated winding. The performance are talked in many aspect, as shown in respective section.

In section II, machines with both type of winding are designed. The combination of pole and slot are evaluated by analytical method and verified by FEM analysis. The harmonic and its influence are discussed by analyzing backEMF (electromotive force), torque ripple, MMF etc. In section III, the output performance evaluation of both machine is made. Efficiency and thermal character are calculated by FEM analysis. In addition, the radical force, the main source of machine vibration, is evaluated in both frequency domain and space domain. Section IV discusses the insulation property, which is an important issue for both type windings in medium voltage application. Taken insulation as the main problem, electrical field analysis is done by FEM analysis to show the difference between both types of machine.

\section{ElectricAl MACHINE Design}

Medium voltage $(4 \mathrm{kV})$ is selected for the generation system considering offshore tidal generation. The requirements of the electrical machine are presented in Table I. The high dc link voltage as mentioned above provides certain advantages. However, winding insulation becomes a challenge. Two different type of winding arrangements are considered: concentrated and distributed overlap winding for design. Fig. 1 presents the side view of the end windings of the two type of windings. It could be seen that the concentrated winding layout is more compact with shorter end windings. Also, the end windings of concentrated 
TABLE I

MAIN REQUIREMENT OF THE MACHINE

\begin{tabular}{ll|l||l|l}
\hline Parameter & Value & Parameter & Value \\
\hline $\begin{array}{l}\text { Nominal Power } \\
(\mathrm{kW})\end{array}$ & 50 & Torque (Nm) & 120 \\
\hline $\begin{array}{l}\text { Maximum Speed } \\
(\mathrm{rpm})\end{array}$ & 4000 & $\begin{array}{l}\text { DC B Bus } \\
\text { voltage (V) }\end{array}$ & 4000 \\
\hline $\begin{array}{l}\text { Outer Diameter } \\
(\mathrm{mm})\end{array}$ & 250 & $\begin{array}{l}\text { Stack Length } \\
(\mathrm{mm})\end{array}$ & 160 \\
\hline
\end{tabular}

winding machine have no overlap between coils in different slots and phases. This reduces the voltage drop between coils and may reduce the insulation requirement greatly. In the traditional distributed overlap winding the coils are twisted in the end winding and is more difficult to insulate.
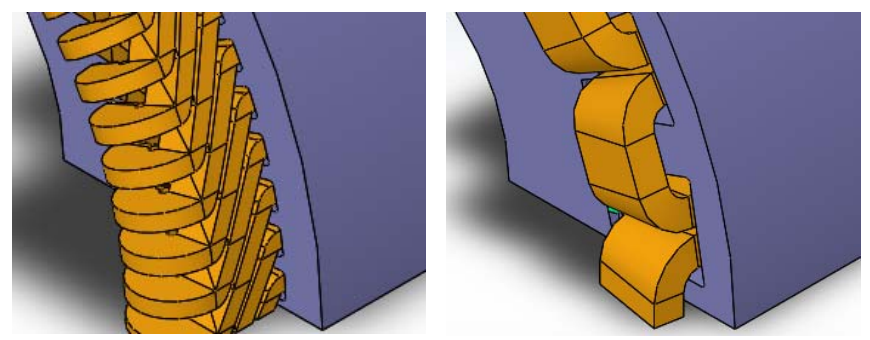

Fig. 1. (a) Distributed winding

(b) Concentrated winding

\section{A. Evaluation of poles and slots combination}

A higher number of pole pairs may be preferred as it results in to thinner yoke and increase torque density of the machine. But, decreasing the thickness of yoke can lead to weak structure of the stator and lower its ability to absorb mechanical vibrations. Furthermore, higher pole-pairs means higher operating frequency for the same rotor speed which increases core loss. Hence, an optimal number of pole pairs is a compromise between torque density, core loss and structural stiffness of the stator. For this work, number of pole pairs is chosen as 6 .

Concentrated winding has its special restricts of poles and slots combination. According to the basic theory of winding, the number of slots per pole of each phase $q$ can be given as

$$
q=\frac{Q}{2 m p}
$$

where $Q$ represents the number of slots, $m$ is the number of phases and $p$ is the number of pole-pairs. In distributed overlap winding, $q$ is an integer, the coil pitch is roughly $m \cdot q$. In concentrated winding combination, $q$ is a fraction and coil pitch is 1. In order to make a comparison between concentrated and distributed overlap winding combination, both kind of winding machine are designed with identical pole-pairs number and rotor topology. For concentrated winding, because of the coil pith restriction the slots number is chosen 18 , whose $q$ is $1 / 2$. The distributed overlap winding slots number is chosen as 72 , whose $q$ is 2 .

Winding factor $K_{d p}$ is a key parameter when choosing between the two types of the winding. It shows the utilization ratio of coil and it is directly proportional to backEMF and output torque. Thus, higher winding factor means better utilization. Furthermore, the winding factor can reduce or cancel some harmonics by designing the machine to have
TABLE II

WINDING FACTOR OF DIFFERENT WINDING

\begin{tabular}{c|c|c||c|c|c}
\hline $\begin{array}{c}\text { Harmonic } \\
\text { order }\end{array}$ & DW & CW & $\begin{array}{c}\text { Harmonic } \\
\text { order }\end{array}$ & DW & CW \\
\hline 1 & 0.933 & 0.866 & 17 & 0.067 & 0.866 \\
\hline 3 & 0.500 & 0.000 & 19 & 0.067 & 0.866 \\
\hline 5 & 0.067 & 0.866 & 21 & 0.500 & 0.000 \\
\hline 7 & 0.067 & 0.866 & 23 & 0.933 & 0.866 \\
\hline 9 & 0.500 & 0.000 & 25 & 0.933 & 0.866 \\
\hline 11 & 0.933 & 0.866 & 27 & 0.500 & 0.000 \\
\hline 13 & 0.933 & 0.866 & 29 & 0.067 & 0.866 \\
\hline 15 & 0.500 & 0.000 & 31 & 0.067 & 0.866 \\
\hline
\end{tabular}

a very small or zero $K_{d p}$ for that particular order of harmonic. Table II shows the winding factor starting from fundamental to $31^{\text {st }}$ harmonic for the two different winding arrangements. Due to the short pitched coils, the distributed winding has a lower winding factor for the 5th and 7 th harmonic which are responsible for torque ripple having 6 times the frequency of fundamental. The winding factor of all harmonic for concentrated winding is the same and it is smaller than distributed winding.

Magnetic motive force (MMF) generated by the current in the coils distributed in different stator slots in space determines the main performance of machine. Different winding arrangements result in different MMF waveform and its harmonic vary largely. With multiphase sinusoidal current flow into the coils arranged in different slots, the coils generate rotating MMF along the stator bore. The fundamental component of MMF interacting with the rotor magnet field generates the output torque. The harmonics of the MMF interact with the rotor field as well, but only generate alternating force on stator instead of output torque. Fig. 2 shows the fast Fourier transform (FFT) result for both type winding arrangements. With roughly the same level of fundamental MMF, the harmonics differ a lot. Concentrated winding has more harmonic because it takes harmonic as fundamental, thus lots of harmonic are excited, that will be talked in detail in subsection B.

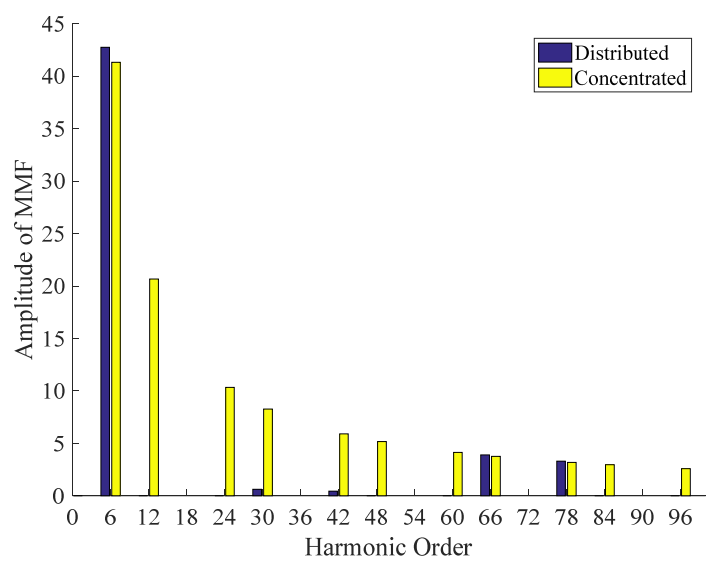

Fig. 2. FFT result of different type winding's MMF.

\section{B. Electromagnetic Design Analysis}

Two electrical machines are designed with different types of winding combination with same rotor topology, yoke 
TABLE III

MAIN PARAMETERS OF THE MACHINE

\begin{tabular}{c|c|c}
\hline & Distributed & Concentrated \\
\hline Number of slots & 72 & 18 \\
\hline Turns per phase & 240 & 300 \\
\hline Coil pitch & 5 & 1 \\
\hline $\begin{array}{c}\text { Phase Resistance } \\
(\mathrm{m} \Omega) 100^{\circ} \mathrm{C}\end{array}$ & 1.447 & 1.624 \\
\hline Yoke thickness $(\mathrm{mm})$ & 10 & 10 \\
\hline Teeth width (mm) & 3.15 & 12.5 \\
\hline Slot fill factor $(\%)$ & 60.7 & 61.8 \\
\hline $\begin{array}{c}\text { Length of end } \\
\text { winding }(\mathrm{mm})\end{array}$ & 68 & 52 \\
\hline Net Weight $(\mathrm{kg})$ & 42.3 & 40.1 \\
\hline
\end{tabular}

thickness, total slot area and slot fill factor. The main parameters of the designed machines are presented in Table III. The concentrated winding has higher number of series turns because of its lower fundamental winding factor. So, the resistance is larger than distributed overlap winding. But concentrated winding has a 30.8\% shorter end winding because of its compact winding structure as shown in fig. 1 . Fig. 3 and Fig.4 present the flux lines and flux density distribution at no load for both designs. The flux density in teeth and yoke isn't that even. The flux density in the yoke of the two machine is the same around $1.1 \mathrm{~T}$ which is far from the saturation value (typically $1.8 \mathrm{~T}$ ). In this design, yoke stiffness is taken more in consideration, also thicker yoke can reduce more vibration, which is generated on the teeth and conducted to the frame via the yoke. The teeth flux density is around $1.4 \mathrm{~T}$, which is in a suitable level at no load The teeth flux density in distributed winding is slightly higher than concentrated winding, even though its total width of teeth per pole is roughly the same. Distributed winding separates concentrated winding's tooth into three, so there is less leakage of rotor and saturation in teeth tip.

\section{1) Slot harmonic canceling}

The slot opening in the stator leads to cogging torque at no load operation and torque ripple at load operation. Both cogging torque and torque ripple deteriorate machine performance. According to electrical machine theory, slot opening generates tooth harmonics, which have the same winding factor with the fundamental wave. That means it

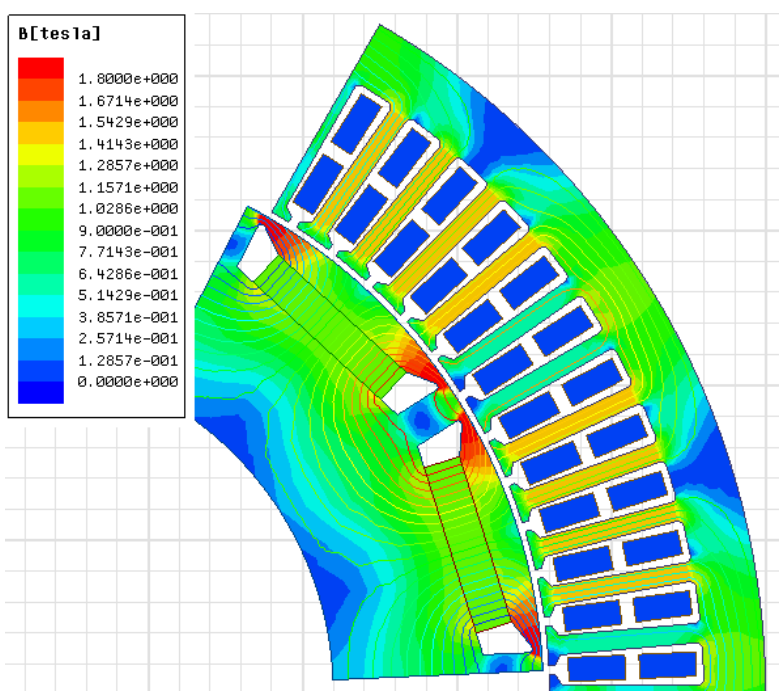

Fig. 3. Flux density distribution of distributed winding at no load.

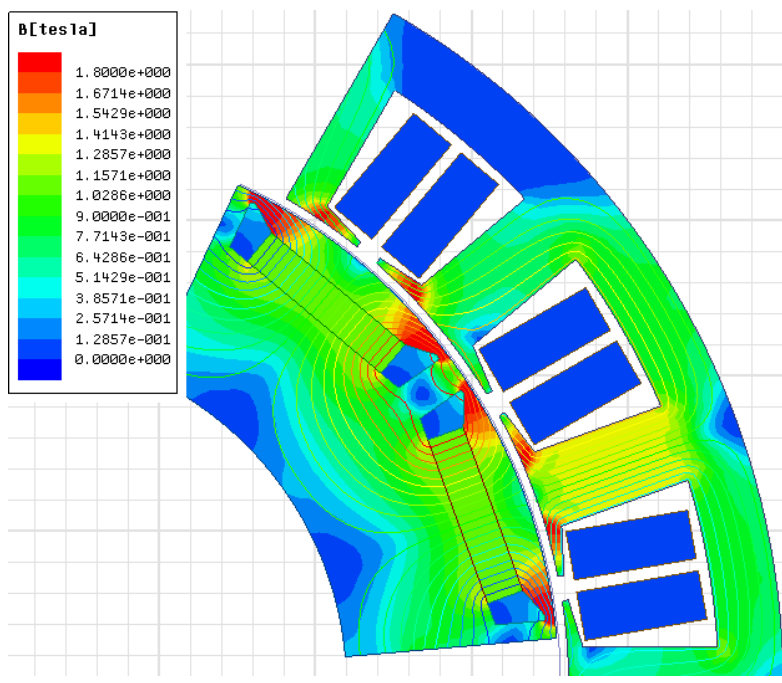

Fig. 4. Flux density distribution of concentrated winding at no load.

can't be canceled by short pitching or other special coil arrangement. In distributed overlap winding case, the order of the slot harmonic is given by (2)

$$
\gamma=2 m q \pm 1
$$

where $m$ is the number of phases, $q$ is the number of slot per pole per phase. If $\mathrm{q}$ is a proper fraction, it is $q=c / d, c$ is numerator of $q, d$ is denominator of $q$. In concentrated winding, the tooth harmonic order is given by (3)

$$
\gamma=2 m c \pm 1
$$

in this concentrated winding $q=1 / 2, c=1$.

Skewing slot or rotor in axial makes a phase shift to both fundamental and harmonics adding the positive and negative peak of the tooth harmonics together. If an appropriate skew angle is chosen, the tooth harmonic can be canceled significantly. The skew of stator slot reduces net slot area and make it hard to manufacture. So, the skewing of rotor is selected to cancel the tooth harmonic. The selection of skew angel $\theta$ of the rotor is given by (4) and (5) for concentrated and distributed windings respectively.

$$
\begin{aligned}
& \theta=\frac{360}{Q} \\
& \theta=\frac{360}{d \cdot Q}
\end{aligned}
$$

in concentrated winding case $q=1 / 2, d=2$. The rotor skew angel for distributed winding with 72 slots and 12 poles is 5 degrees. Similarly, for concentrated winding with 18 slots and 12 poles it is 10 degrees. The rotor is skewed with the calculated angle and cogging torque and torque ripple is analyzed. The cogging torque is shown in Fig. 5, the peak to peak value is $2.47 \mathrm{Nm}$ and $1.53 \mathrm{Nm}$ for distributed winding and concentrated winding respectively which is low compare to the rated torque defined in Table I. The concentrated winding has, lower cogging torque because of the arrangement of stator slots and rotor poles. Fig. 6 shows the output torque and torque ripple for both type of windings. With the same value current supply (peak amplitude 17A), distributed winding output torque is $118.8 \mathrm{Nm}$, and the torque ripple is $2.62 \%$. For concentrated winding, it is 121.8 $\mathrm{Nm}$ and $2.38 \%$ respectively. Both designs have a comparable performance in terms of output torque with concentrated winding having $2.53 \%$ more output torque. 


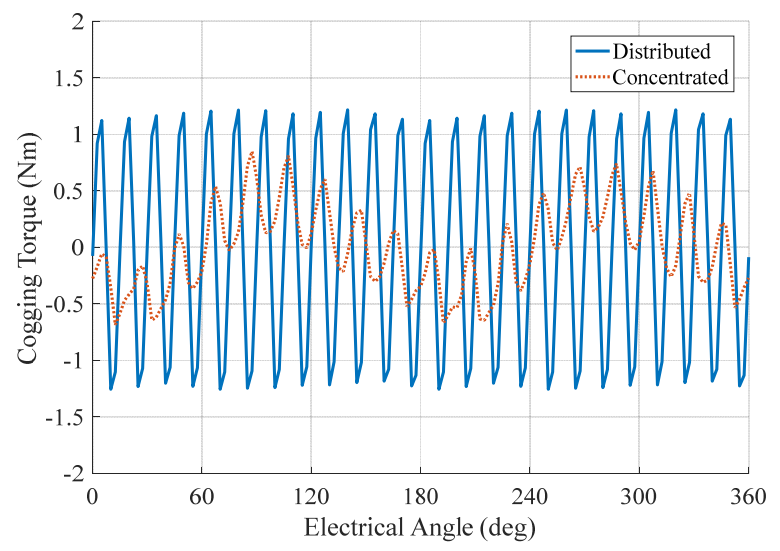

Fig. 5. Cogging torque of both machine.

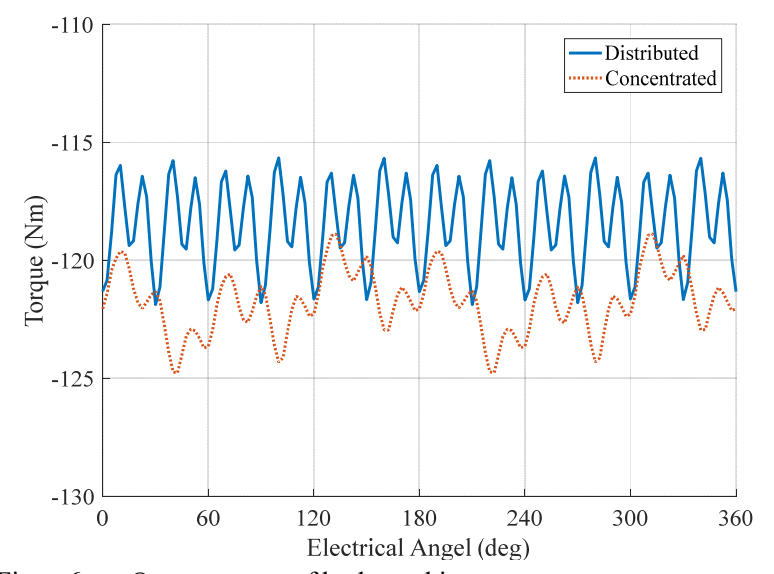

Fig. 6. Output torque of both machine.

\section{2) Electromagnetic Performance}

As presented in Table II, the fundamental winding factor is different for the two machines. So, appropriate series number of turns per phase is selected to obtain approximately the same back-EMF at no load for the two machines. Fig. 7 shows the per phase back-EMF of the two type of windings and as seen they have approximately the same amplitude. The peak value and the RMS value of the distributed overlap winding back-EMF is $1681.2 \mathrm{~V}$ and $1321.1 \mathrm{~V}$. And for the concentrated winding back-EMF is $1766.8 \mathrm{~V}$ and $1253.1 \mathrm{~V}$. Because of the distributed arrangement of coils the back-EMF wave have a flat waveform.

Harmonic analysis of back-EMF is implemented in Fig. 8. It is obvious that distributed winding has a significant $3 \mathrm{rd}$ harmonic and 9th harmonics. But if $\mathrm{n}$ is an integer, then harmonics with order $3 \mathrm{n}$ can be canceled by star connection of three phase windings. What should pay close attention to is the slot harmonics. From the FFT result no significant slot harmonics can be found. This illustrated the skewing of rotor works well on slot harmonic cancelling. The total harmonic distortion (THD) is defined as the ratio of the sum of the powers of all harmonic components to the power of the fundamental frequency. It is widely used to evaluate the waveform. The THD of the back-EMF are $1.79 \%$ and $1.32 \%$ for distributed winding and concentrated winding respectively. They are almost at the same level, but concentrated winding having a slightly lower THD. Because lots of harmonics are cancelled by rotor skewing.

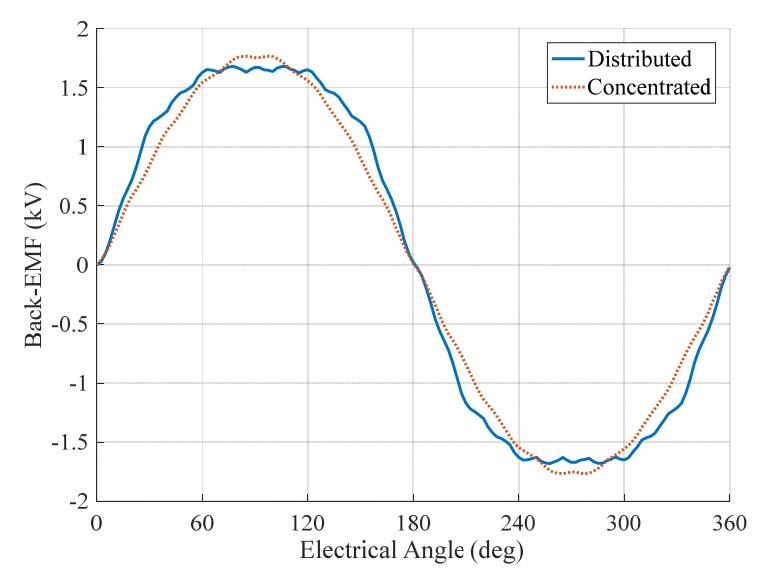

Fig. 7. Back-EMF curve of different type winding.

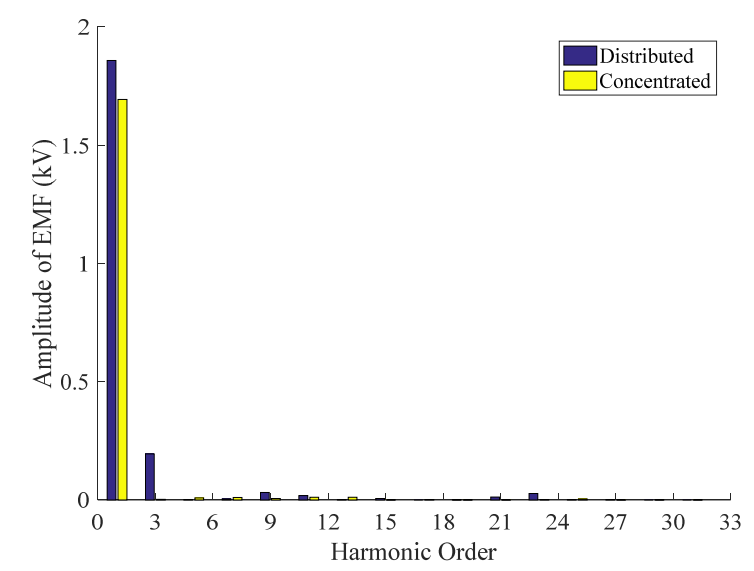

Fig. 8. FFT result of back-EMF

\section{Electromagnetic Force Analysis}

Current flowing into the stator winding interacts with rotor magnetic field produced by permanent magnets to generate electromagnetic force. The tangential force on the rotor and stator is responsible for output torque and torque ripple, whereas the radical force on the stator and rotor does not generate any output power. And, the value of the radial force is larger roughly 10 times than the tangential force. Furthermore, the radial force generates vibration which is the main source of high frequency acoustic noise of the machine. If the space order of the vibration mode on the stator is lower than 5 , then the vibration has a huge amplitude and can cause fatigue of mechanical structure. The electromagnetic radial force on the stator both in frequency domain and space domain is analyzed using FEM simulation. The machine operated at 4000rpm, peak amplitude 17A current supply.

\section{1) Frequency domain analysis}

According to Maxwell stress tensor equation, the electromagnetic radical force density is given by (6).

$$
\mathrm{F}_{\mathrm{n}}=\frac{B_{n}^{2}-B_{t}^{2}}{2 \mu_{0}}
$$

where $B_{n}$ is the radial flux density in the air gap, $B_{t}$ is the tangential flux density in the air gap. $\mu_{0}$ is vacuum permeability which is a constant number which equals to $4 \pi \times 10^{-7} \mathrm{H} / \mathrm{m}$. In frequency domain, the lower harmonic order vibration is easy to reduce or damp. The high frequency vibration generates high frequency acoustic noise 


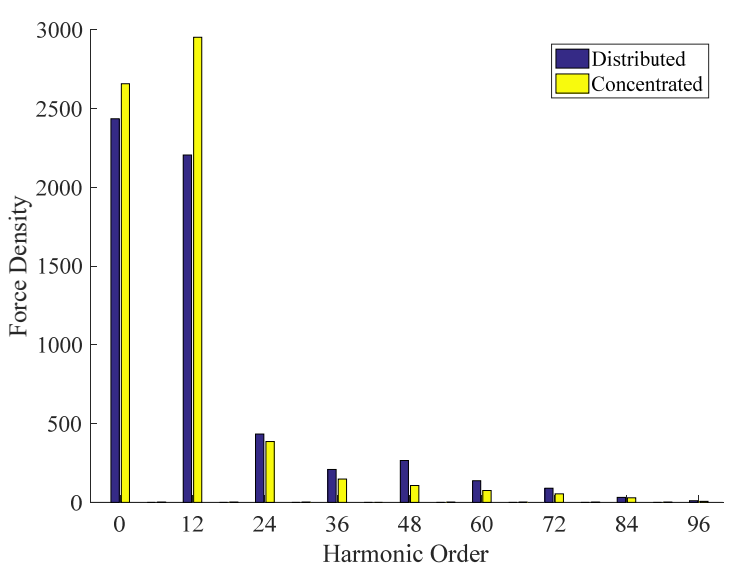

Fig. 9. Frequency domain FFT of force density on tooth.

which is very annoying to the environment. Even its amplitude is not that high, but human ears are very sensitive to high frequency acoustic noise. The force on a tooth is calculated and the FFT of the force in an electrical period is show in Fig. 9. The order 0 is the DC component of the radical force, which has no influence on vibration. The order of harmonics in the radial force for both type of winding is almost same. The amplitude the radial force at high frequency (24th, 36th, 48th, 60th, and 72th) is a little higher than concentrated winding.

2) Space domain analysis

The electromagnetic force density in the air gap is

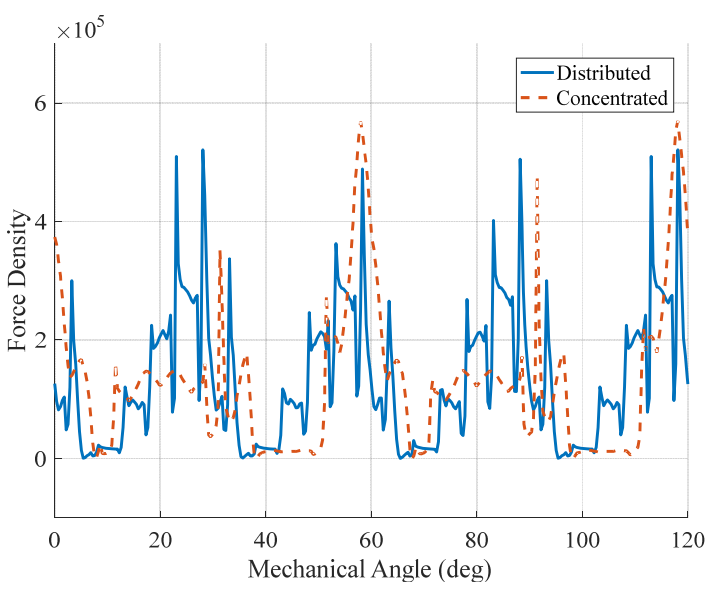

Fig. 10. Space force density along the air gap.

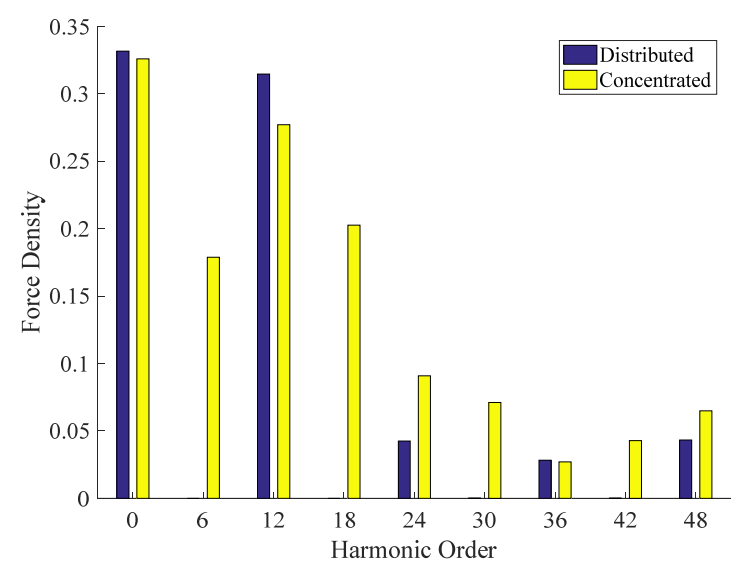

Fig. 11. Space domain FFT of force density in air gap. calculated based on (6) by FEM simulations. The force density along the stator bore is present in Fig. 10. Because of its repeating, the curve is only presents in a third of the stator bore circumference. It could be observed that both type windings have 4 sharp peaks having similar peak amplitude. But the concentrated winding's 4 peaks are not at the same amplitude. In the curve the sharply drop down is caused by the slot open. Fig. 11 shows the FFT result of force density along the stator bore. The space order 0 has no significant influence on vibration, because of its breathing mode in space. The lower order space harmonics of the radial force generates larger vibrations on stator bore, unlike in frequency domain where the lower order harmonics of the force can be easily damped, the lower order space harmonics generates higher amplitude vibration. The lowest order of the space harmonics of the radial force for 12 and 6 for distributed winding and concentrated winding respectively. This means that the amplitude of vibration generated by radical force in concentrated winding is larger than in distributed winding. But the lowest order of concentrated winding is higher than 5 and so it could still be acceptable.

\section{EFFICIENCY \& THERMAL PERFORMANCE}

Table IV shows the efficiency data of both the machines operated at 4000rpm, peak amplitude 17A phase current input, with maximum torque per ampere (MTPA) control for selecting the operating point. The core loss is obtained from FEM calculation using ANSYS Maxwell.

TABLE IV

EFFICIENCY AND LOSSES OF THE MACHINE

\begin{tabular}{c|c|c}
\hline & Distributed & Concentrated \\
\hline Output Torque $(\mathrm{Nm})$ & -118.8 & -121.8 \\
\hline Peak Current $(\mathrm{A})$ & 17 & 17 \\
\hline $\begin{array}{c}\text { Torque Factor } \\
(\mathrm{Nm} / \mathrm{A})\end{array}$ & 9.88 & 10.13 \\
\hline $\begin{array}{c}\text { Power Density } \\
(\mathrm{kW} / \mathrm{kg})\end{array}$ & 1.14 & 1.24 \\
\hline $\begin{array}{c}\text { Current Density } \\
(\mathrm{A} / \mathrm{mm})\end{array}$ & 5.2 & 6.8 \\
\hline Copper Loss $(\mathrm{W})$ & 627.4 & 704.3 \\
\hline Core Loss $(\mathrm{W})$ & 865.9 & 914.7 \\
\hline Efficiency $(\%)$ & 96.34 & 96.20 \\
\hline
\end{tabular}

The torque factor, defined as output torque divided by input stator current (RMS value), can be used to evaluate the output torque ability of an electrical machine. The concentrated winding's torque factor is $2.53 \%$ higher than the distributed overlap winding indicating a better torque producing ability. Due to the lower fundamental winding factor, the copper loss and current density of the concentrated winding is higher than distributed winding. The total efficiency of the machine however is almost the same. More harmonic components exist in the MMF as shown in section II, leading to higher core loss in concentrated winding machine. Fig. 12 shows the cross-section view of the machine structure.

Water is used as the coolant. The water jacket channel is spiral, and the temperature of the water at the inlet is assumed to be $15{ }^{\circ} \mathrm{C}$. The flow rate is $12 \mathrm{~L} / \mathrm{min}$. Based on the losses in Table III, the thermal performance is calculated. 


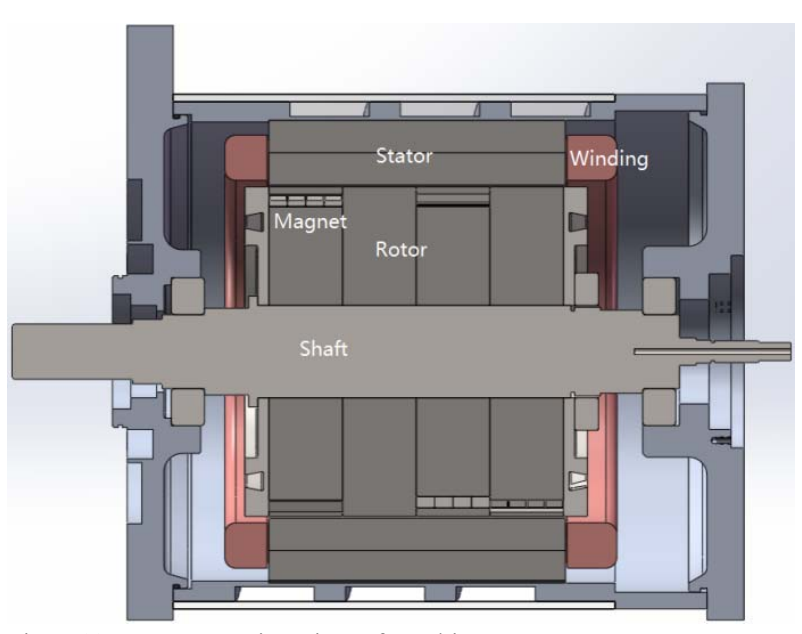

Fig. 12. Cross-section view of machine.

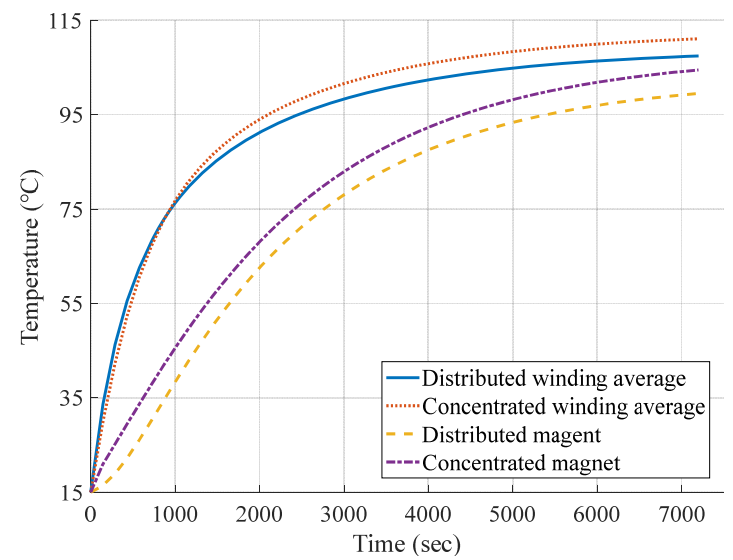

Fig. 13. Winding and magnet thermal curve of different machine.

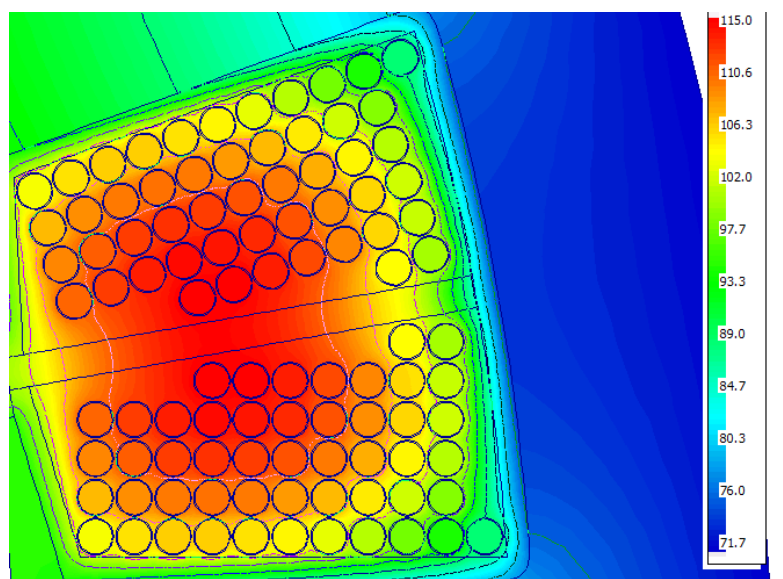

Fig. 14. Temperature distribution in slot of concentrated winding.

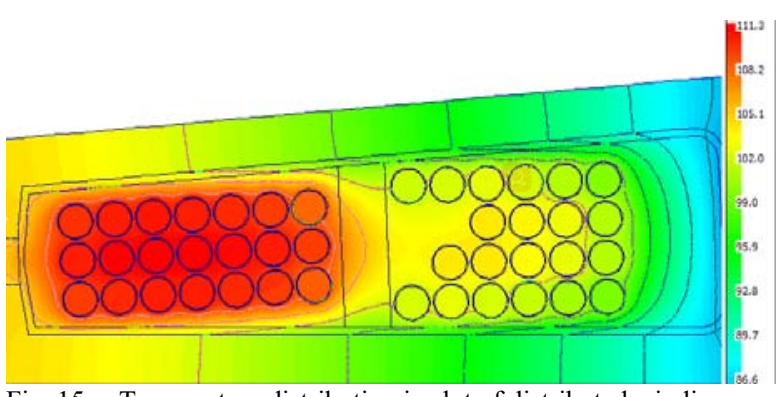

Fig. 15. Temperature distribution in slot of distributed winding.

The machine operated for 2 hours at $4000 \mathrm{rpm} 17$ A phase current. The average temperature curve of winding and magnet is presented in Fig. 13. Because of the higher MMF harmonics in concentrated winding the eddy loss in magnet is higher, thus the temperature is higher than distributed winding. The magnets of both machines are separated into 16 pieces in axial to decrease the eddy loss. So the temperature of both magnet didn't differ that much. The temperature distribution in slots is shown respectively in Fig. 14 and Fig. 15. Due to larger slot area of concentrated winding combination, the heat is more concentrated in the middle of the slot.

\section{INSULATION ANALYSIS}

This section discusses the insulation of the two type of windings, which is a challenge for this application. In distributed winding, the wires of each coil are placed in stator slots to obtain a spatial distribution along the air gap close to an ideal sine wave. For small machines made with round enameled wires, the exact relative position of each turn in a slot is difficult to define precisely and varies from one machine to the next. The winding is not deterministic; it has random properties. The probability to have the input turn adjacent to the output one can't be neglected. In that case, the full coil voltage is imposed to the turn-to-turn insulation made of two thin polymer layers and can cause partial discharge (PD). Concentrated winding has a separated winding structure as shown in Fig. 1. Furthermore, its end winding has no overlap, avoiding possibilities of discharge between phases. The FEM analysis of electrical field is made in one slot which have two phase winding arranged inside. The insulation material and structure of insulation material arrangement in slots is the same. Fig.16 and Fig.17 show the electrical field distribution in slot between the two layers of winding. In the case of distributed winding, the highest electric field exceeds $70 \mathrm{kV} / \mathrm{cm}$ which may cause discharge. In concentrated winding case however, it is $22 \mathrm{kV} / \mathrm{cm}$ below the safety range $(35 \mathrm{kV} / \mathrm{cm})$ of the insulation material.

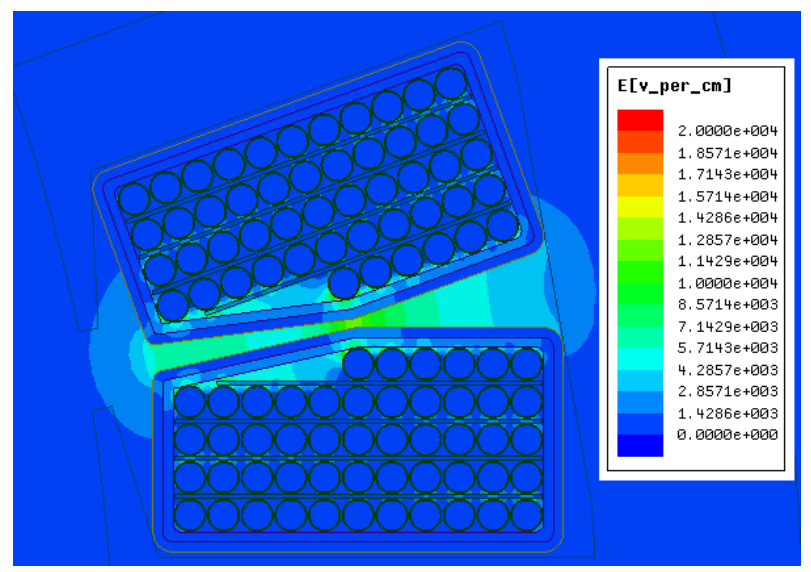

Fig. 16. Electrical field distribution in slot of concentrated winding.

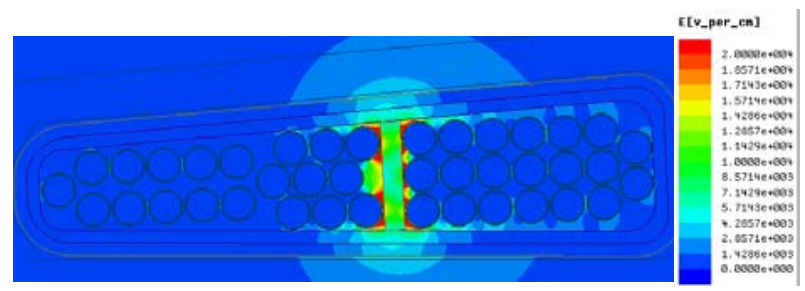

Fig. 17. Electrical field distribution in slot of distributed winding. 


\section{CONCLUSION}

The design of an interior permanent magnet generator for medium voltage tidal power conversion is analyzed using two different type of winding arrangements. The results show that the concentrated winding machine has roughly the same performance. The concentrated winding is observed to have slightly lower harmonics in the back-EMF resulting into lower torque ripple and lower cogging torque. And, the distributed overlap winding a little better performance in efficiency, thermal performance and may have lower vibrations. But when it comes to the medium voltage, the concentrated winding have a great benefit in insulation, easy manufacturing and material saving. All the results show a great promising future of concentrated winding applied in medium voltage area.

\section{REFERENCES}

[1] D.O'sullivan A. Lewis "Generator selection and comparative performance in offshore oscillating water column ocean wave energy converters" IEEE Trans. Energy Convers. vol. 26 no. 2 pp. 603-614 Jun. 2011.

[2] U. Kim and D. K. Lieu, "Magnetic field calculation in permanent magnet motors with rotor eccentricity: With slotting effect considered," IEEE Trans. Magn., vol. 34, no. 4, pp. 2253-2266, Jul. 1998.

[3] D.Zarko, T.A.Lipo, and D.Ba"Analytical calculation of magnetic field distribution in the slotted air gap of a surface permanent-magnet motor using complex relative air-gap permeance," IEEE Trans. Magn., vol. 42,no. 7, pp. 1828-1837, Jul. 2006.

[4] L. Zhu, S. Z. Jiang, Z. Q. Zhu, and C. C. Chan, "Analytical methods for minimizing cogging torque in permanent-magnet machines," IEEE Trans. Magn., vol. 45, no. 4, pp. 2023-2031, Apr. 2009.

[5] D. Zarko, D. Ban, and T. A. Lipo, "Analytical solution for cogging torque in surface permanent-magnet motors using conformal mapping," IEEE Trans. Magn., vol. 44, no. 1, pp. 52-65, Jan. 2008.

[6] D.Zarko, D.Ban, and T.A.Lipo, "Analytical solution for electromagnetic torque in surface permanent-magnet motors using conformal mapping," IEEE Trans. Magn., vol. 45, no. 7, pp. $2943-$ 2954, Jul. 2009.

[7] G. Dajaku and D. Gerling, "The influence of permeance effect on the magnetic radial forces of permanent magnet synchronous machines," IEEE Trans. Magn., vol. 49, no. 6, pp. 2953-2966, Jun. 2013.
[8] M. Valavi, A. Nysveen, R. Nilssen, and T. Rølvåg, "Slot harmonic effect on magnetic forces and vibration in low-speed permanentmagnet machine with concentrated windings," IEEE Trans. Ind. Appl., vol. 50, no. 5, pp. 3304-3313, Sep./Oct. 2014.

[9] J. Pyrhönen, H. Jussila, Y. Alexandrova, P. Rafajdus, and J. Nerg, "Harmonic loss calculation in rotor surface permanent magnets-New analytic approach," IEEE Trans. Magn., vol. 48, no. 8, pp. 23582366,Aug. 2012

[10] E. Fornasiero, N. Bianchi, and S. Bolognani, "Slot harmonic impact on rotor losses in fractional-slot permanent-magnet machines," IEEE Trans. Ind. Electron., vol. 59, no. 6, pp. 2557-2564, Jun. 2012.

\section{BIOGRAPHIES}

Jian Zhao was born in China, on April 29, 1988. He received his master degree in electrical engineering from Harbin Institute of Technology, Harbin China, in 2013. He was working in electrical machine applied in industrial automation and electric vehicle from 2013 to 2017. He is currently a researcher in electric power engineering division at Chalmers University of Technology science 2017. His research interest include electrical machine design in renewable energy system and electrical vehicle.

Yujing Liu received B.Sc., M.Sc. and Ph.D. degrees in electrical engineering from Harbin Institute of Technology, Harbin, China, in 1982, 1985, and 1988, respectively. In 1996-2013, he worked in ABB Corporate Research, Västerås, Sweden. Since 2013, he is professor on electrical power engineering in Chalmers University of Technology. Gothenburg, Sweden. His interest includes research on motors, converters, and wireless charging for electric vehicles, generators and power electronics for tidal power conversion, and high efficiency machines for energy saving in industrial applications. Yujing Liu is senior IEEE member and member in Swedish Standard Committee on Electrical Machines.

Xiangdong $\mathrm{Xu}$, received the M.Sc. degree in electrical engineering and Ph.D. degree in high voltage engineering from Chalmers University of Technology, Gothenburg, Sweden in 2009 and 2015. In 2010, He was working with grid asset management in the University of Southern Denmark. He is currently employed as a researcher in electric power engineering division at Chalmers University of Technology, where his main interests are high voltage engineering and dielectric characterization techniques. 